\title{
Spectral Direction Splitting Schemes for the Incompressible Navier-Stokes Equations
}

\author{
Lizhen Chen $^{1}$, Jie Shen ${ }^{1,2}$ and Chuanju $\mathrm{Xu}^{*, 1}$ \\ ${ }^{1}$ School of Mathematical Sciences, Xiamen University, 361005 Xiamen, China. \\ ${ }^{2}$ Department of Mathematics, Purdue University, West Lafayette, IN, 47907, USA. \\ Received 19 April 2011; Accepted (in revised version) 24 May 2011
}

Available online 27 July 2011

\begin{abstract}
We propose and analyze spectral direction splitting schemes for the incompressible Navier-Stokes equations. The schemes combine a Legendre-spectral method for the spatial discretization and a pressure-stabilization/direction splitting scheme for the temporal discretization, leading to a sequence of one-dimensional elliptic equations at each time step while preserving the same order of accuracy as the usual pressurestabilization schemes. We prove that these schemes are unconditionally stable, and present numerical results which demonstrate the stability, accuracy, and efficiency of the proposed methods.
\end{abstract}

Key words: Navier-Stokes equations, projection method, direction splitting, spectral methods.

\section{Introduction}

A main difficulty in solving the incompressible Navier-Stokes equations is how to deal with the divergence-free constraint which couples the velocity and the pressure. There exists an enormous amount of literature on this subject. A popular and efficient approach is to use a projection type method which was originated from the pioneering works of Chorin [2] and Temam [9]. This type of methods decouples the computation of pressure from that of velocity, and only requires to solve a sequence of Poisson type equations at each time step. We refer to [5] for an overview of the projection type methods.

Recently, Guermond and Minev [4] (see also [6]) proposed to combine the pressurestabilization method (cf. [3,10]) and the direction splitting technique [7] for the time discretization of the incompressible Navier-Stokes equations, leading to a sequence of one-dimensional problems at each time step. In [4] and [6], it is shown that these semi-discretized pressure-stabilization/direction splitting schemes are unconditionally stable and have the same order of accuracy as the usual projection schemes without spatial discretization.

${ }^{*}$ Corresponding author. Email address: cjxu@xmu.edu.cn (C. Xu) 
In this paper, we consider the stability of the spatial discretization of the pressurestabilization/direction splitting schemes by using a Legendre-spectral method. It turns out that a usual Legendre-Galerkin or Legendre-collocation method will not lead to unconditionally stable schemes as in the semi-discretized case. To overcome this difficulty, we construct a hybrid of Legendre-Galerkin and Legendre-collocation methods which is easy to implement and enables us to prove the unconditional stability. We also construct a pressure-stabilization/direction splitting scheme for problems with variable viscosity and prove that a semi-discretized version is unconditionally stable.

The outline of the paper is as follows. In the next section we construct a fully discretized direction splitting scheme by using a hybrid of Legendre-collocation and Legendre-Galerkin methods. In Section 3, we carry out a stability analysis for the fully discretized schemes for both the standard and rotational versions. We present in Section 4 a generalization of the spectral direction splitting scheme to the Navier-Stokes equations with variable viscosity and prove a stability result in a simplified semi-discrete case. We present numerical results and discussions in Section 5.

\section{Spectral Direction Splitting Schemes}

The direction splitting schemes are usually applied to separable domains. We shall restrict our attention in this paper to $\Omega=(-1,1)^{2}$, and consider the time-dependent NavierStokes equations:

$$
\begin{cases}\frac{\partial \boldsymbol{u}}{\partial t}-\boldsymbol{v} \Delta \boldsymbol{u}+\boldsymbol{u} \cdot \nabla \boldsymbol{u}+\nabla p=\boldsymbol{f}, & \text { in } \Omega \times(0, T], \\ \nabla \cdot \boldsymbol{u}=0, & \text { in } \Omega \times[0, T], \\ \left.\boldsymbol{u}\right|_{\partial \Omega}=\mathbf{0}, & \text { in }[0, T], \\ \left.\boldsymbol{u}\right|_{t=0}=\boldsymbol{u}_{0}, & \text { in } \Omega,\end{cases}
$$

where $v$ is the viscosity coefficient, $\boldsymbol{u}$ and $p$ stand for the velocity vector and the pressure respectively. Since the treatment of the nonlinear term does not have an essential impact on the pressure-stabilization and direction splitting. We shall restrict our attention in this paper to the Stokes case, i.e., (2.1) without the nonlinear term.

\subsection{Direction splitting scheme}

We start with a second-order pressure-stabilization scheme:

$$
\left\{\begin{array}{l}
\frac{1}{\triangle t}\left(\boldsymbol{u}^{n+1}-\boldsymbol{u}^{n}\right)-v \Delta \frac{\boldsymbol{u}^{n+1}+u^{n}}{2}+\nabla p^{n}=\boldsymbol{f}^{n+\frac{1}{2}}, \quad \text { in } \Omega, \\
\left.\boldsymbol{u}^{n+1}\right|_{\partial \Omega}=0,
\end{array}\right.
$$




$$
\begin{aligned}
& \left\{\begin{array}{l}
\frac{1}{\Delta t} \nabla \cdot \boldsymbol{u}^{n+1}+\Delta \phi^{n+1}=0, \text { in } \Omega, \\
\left.\frac{\partial \phi^{n+1}}{\partial \boldsymbol{n}}\right|_{\partial \Omega}=0,
\end{array}\right. \\
& p^{n+1}=\phi^{n+1}+p^{n}-\chi v \nabla \cdot \boldsymbol{u}^{n+1} .
\end{aligned}
$$

The above scheme with $\chi=0$ was studied in [8], and it was shown that the scheme is second-order accurate for the velocity and first-order accurate for the pressure in the $L^{2}$ norm. The case with $0<\chi \leq 1$ is related to the scheme introduced in [10], and it can be shown that the scheme is second-order accurate for the velocity and 3/2-order accurate for the pressure in the $L^{2}$-norm. We shall adopt the classification in [5] and refer the case with $\chi=0$ as the standard form and the case with $0<\chi \leq 1$ as the rotational form.

While it is easy to write down a direction splitting (or the ADI) scheme for the step (2.2), how to deal with (2.3) is not obvious. It was observed in [4] that the order of accuracy for the scheme (2.2)-(2.4) remains the same if the Laplace operator in (2.3) is replaced by a more general operator $A$ defined by a bilinear form $a(\cdot, \cdot)$ satisfying the following properties:

$$
\begin{aligned}
& a(\cdot, \cdot) \text { is symmetric, and }\|\nabla \psi\|_{L^{2}}^{2} \leq a(\psi, \psi), \quad \forall \psi \in D(A), \\
& a(\phi, \psi)=\int_{\Omega} \psi A \phi d x, \quad \forall \phi, \psi \in D(A),
\end{aligned}
$$

where $D(A)$ is the domain of $A$.

The operator $A$ used in [4] is the following:

$$
A:=\left(1-\partial_{x x}\right)\left(1-\partial_{y y}\right),
$$

with the domain

$$
D(A):=\left\{\psi,\left(1-\partial_{y y}\right) \psi, A \psi \in L^{2}(\Omega),\left.\partial_{y} \psi\right|_{y= \pm 1}=0,\left.\partial_{x}\left(\left(1-\partial_{y y}\right) \psi\right)\right|_{x= \pm 1}=0\right\} .
$$

The associated bilinear form $a(\cdot, \cdot)$ is given by

$$
a(\phi, \psi)=(\phi, \psi)+(\nabla \phi, \nabla \psi)+\left(\partial_{x y} \phi, \partial_{x y} \psi\right),
$$

where $(\cdot, \cdot)$ means the standard $L^{2}$-inner product. It is an easy matter to verify that such a $A$ and $a(\cdot, \cdot)$ satisfy the property $(2.5)$.

The following direction splitting scheme is proposed in [4].

Setting $\boldsymbol{u}^{0}=\left.\boldsymbol{u}\right|_{t=0}, p^{-\frac{1}{2}}=\left.p\right|_{t=0}$ and $\phi^{-\frac{1}{2}}=0$.

For $n \geq 0$, we look for $\left(\boldsymbol{u}^{n+\frac{1}{2}}, \boldsymbol{u}^{n+1}, p^{n+\frac{1}{2}}\right)$ as follows:

- Velocity splitting:

$$
\left\{\begin{array}{l}
\frac{\boldsymbol{u}^{n+\frac{1}{2}}-\boldsymbol{u}^{n}}{\frac{1}{2} \Delta t}-v\left(\partial_{x x} \boldsymbol{u}^{n+\frac{1}{2}}+\partial_{y y} \boldsymbol{u}^{n}\right)+\nabla p^{*, n+\frac{1}{2}}=\boldsymbol{f}^{n+\frac{1}{2}}, \\
\left.\boldsymbol{u}^{n+\frac{1}{2}}\right|_{x= \pm 1}=\mathbf{0}
\end{array}\right.
$$




$$
\left\{\begin{array}{l}
\frac{\boldsymbol{u}^{n+1}-\boldsymbol{u}^{n+\frac{1}{2}}}{\frac{1}{2} \Delta t}-v\left(\partial_{x x} \boldsymbol{u}^{n+\frac{1}{2}}+\partial_{y y} \boldsymbol{u}^{n+1}\right)+\nabla p^{*, n+\frac{1}{2}}=f^{n+\frac{1}{2}} \\
\left.\boldsymbol{u}^{n+1}\right|_{y= \pm 1}=\mathbf{0}
\end{array}\right.
$$

where

$$
p^{*, n+\frac{1}{2}}=p^{n-\frac{1}{2}}+\phi^{n-\frac{1}{2}}
$$

- Pressure splitting:

$$
\begin{array}{ll}
\psi^{n+\frac{1}{2}}-\partial_{x x} \psi^{n+\frac{1}{2}}=-\frac{\nabla \cdot u^{n+1}}{\Delta t}, & \left.\partial_{x} \psi^{n+\frac{1}{2}}\right|_{x= \pm 1}=0 \\
\phi^{n+\frac{1}{2}}-\partial_{y y} \phi^{n+\frac{1}{2}}=\psi^{n+\frac{1}{2}}, & \left.\partial_{y} \phi^{n+\frac{1}{2}}\right|_{y= \pm 1}=0
\end{array}
$$

and

$$
p^{n+\frac{1}{2}}=p^{n-\frac{1}{2}}+\phi^{n+\frac{1}{2}}-\chi v \nabla \cdot\left(\frac{1}{2}\left(\boldsymbol{u}^{n+1}+\boldsymbol{u}^{n}\right)\right) .
$$

We observe that each of the substeps (2.8), (2.9), (2.11) and (2.12) is a sequence of onedimensional elliptic problems. Therefore, the overall scheme is extremely efficient. It has been shown in $[4,6]$ that the above scheme is unconditionally stable and preserve the temporal accuracy of the scheme (2.2)-(2.4).

\subsection{Full discretization by a Legendre-spectral method}

We now construct a full discretization scheme by using a Legendre-spectral method which would preserve the stability of the semi-discretized scheme (2.8)-(2.13).

It turns out that a straightforward spatial discretization of (2.8)-(2.13) by using a Legendre-Galerkin method or a Legendre-collocation method does not preserve the unconditional stability of the semi-discretized scheme (2.8)-(2.13). Therefore, we shall construct a hybrid of the Legendre-Galerkin and Legendre-collocation methods which will allow us to prove the unconditional stability while preserving the time accuracy.

We now introduce some basic notations which will be used hereafter. Let $\mathbb{N}$ be the set of all non-negative integers and $\Lambda=(-1,1)$. For any $N \in \mathbb{N}$, we denote by $\mathbb{P}_{N}(\Lambda)$ the set of all polynomials of degree $\leq N$ defined in $\Lambda$, and set $\mathbb{P}_{N}^{0}(\Lambda):=\left\{\phi \in \mathbb{P}_{N}(\Lambda): \phi( \pm 1)=0\right\}$, $\mathbb{P}_{N}(\Omega)=\mathbb{P}_{N}(\Lambda) \otimes \mathbb{P}_{N}(\Lambda), \mathbb{P}_{N}^{0}(\Omega):=\left\{\phi \in \mathbb{P}_{N}(\Omega):\left.\phi\right|_{\partial \Omega}=0\right\}$.

Let $x_{i}$ and $\omega_{i}$ or $y_{j}$ and $\omega_{j}$ for $0 \leq i, j \leq N$ be the nodes and associated weights of the Legendre-Gauss-Lobatto quadrature in $\Lambda$. Let $\Sigma$ be the set of all collocation points $\left(x_{i}, y_{j}\right)_{i, j=0}^{N}$, and $\Sigma_{I}$ the set of all interior collocation points, i.e. $\Sigma_{I}:=\left\{\boldsymbol{x}_{i j}=\left(x_{i}, y_{j}\right), 1 \leq\right.$ $i, j \leq N-1\}$. We denote by $I_{N}$ the polynomial interpolation operator based on the set $\Sigma$. We define the discrete inner product:

$$
(u, v)_{N, \Omega}:=\sum_{i, j=0}^{N} u\left(\boldsymbol{x}_{i j}\right) v\left(\boldsymbol{x}_{i j}\right) \omega_{i} \omega_{j}, \forall u, v \in C^{0}(\bar{\Omega})
$$


and its associated norm

$$
\|v\|_{N, \Omega}:=(v, v)_{N, \Omega}^{1 / 2}
$$

It is well known (cf. [1]) that

$$
\left\|u_{N}\right\|_{L^{2}} \leq\left\|u_{N}\right\|_{N, \Omega} \leq \sqrt{3}\left\|u_{N}\right\|_{L^{2}}, \forall u_{N} \in \mathbb{P}_{N}(\Omega) .
$$

For any fixed $y \in \Lambda$, we define the discrete scalar product with respect to $x$ direction, $(\cdot, \cdot)_{x, N, \Lambda}$, by

$$
(u, v)_{x, N, \Lambda}:=\sum_{i=0}^{N} u\left(x_{i}, y\right) v\left(x_{i}, y\right) \omega_{i}, \forall u, v \in C^{0}(\bar{\Omega}) .
$$

The notation $(\cdot, \cdot)_{y, N, \Lambda}$ can be defined similarly.

In cases where no confusion would arise, $\Omega$ and $\Lambda$ may be dropped from the notations.

We consider the following full discretized version of the scheme (2.8)-(2.13).

Setting $\boldsymbol{u}_{N}^{0}=\left.I_{N} \boldsymbol{u}\right|_{t=0}, p_{N}^{-\frac{1}{2}}=\left.I_{N} p\right|_{t=0}$ and $\phi_{N}^{-\frac{1}{2}}=0$. For $n \geq 0$, we look for $\left(\boldsymbol{u}_{N}^{n+\frac{1}{2}}, \boldsymbol{u}_{N}^{n+1}\right.$, $\left.p_{N}^{n+\frac{1}{2}}\right)$ as follows:

- Velocity splitting:

Find $\boldsymbol{u}_{N}^{n+\frac{1}{2}} \in \mathbb{P}_{N}(\Omega)$ such that for each $j \in\{0,1, \cdots, N\}, \boldsymbol{u}_{N}^{n+\frac{1}{2}}\left(\cdot, y_{j}\right)$ satisfies

$$
\begin{aligned}
& \frac{\boldsymbol{u}_{N}^{n+\frac{1}{2}}\left(x_{i}, y_{j}\right)-\boldsymbol{u}_{N}^{n}\left(x_{i}, y_{j}\right)}{\frac{1}{2} \Delta t}-v\left(\partial_{x x} \boldsymbol{u}_{N}^{n+\frac{1}{2}}\left(x_{i}, y_{j}\right)+\partial_{y y} \boldsymbol{u}_{N}^{n}\left(x_{i}, y_{j}\right)\right) \\
& \quad+\nabla p_{N}^{*, n+\frac{1}{2}}\left(x_{i}, y_{j}\right)=\boldsymbol{f}^{n+\frac{1}{2}}\left(x_{i}, y_{j}\right), \quad i=1,2, \cdots, N-1, \\
& \boldsymbol{u}_{N}^{n+\frac{1}{2}}\left( \pm 1, y_{j}\right)=\mathbf{0} ;
\end{aligned}
$$

Find $\boldsymbol{u}_{N}^{n+1} \in \mathbb{P}_{N}^{0}(\Omega)$ such that for each $i \in\{1,2, \ldots, N-1\}, \boldsymbol{u}_{N}^{n+1}\left(x_{i}, \cdot\right)$ satisfies

$$
\begin{aligned}
& \frac{\boldsymbol{u}_{N}^{n+1}\left(x_{i}, y_{j}\right)-\boldsymbol{u}_{N}^{n+\frac{1}{2}}\left(x_{i}, y_{j}\right)}{\frac{1}{2} \Delta t}-v\left(\partial_{x x} \boldsymbol{u}_{N}^{n+\frac{1}{2}}\left(x_{i}, y_{j}\right)+\partial_{y y} \boldsymbol{u}_{N}^{n+1}\left(x_{i}, y_{j}\right)\right) \\
& \quad+\nabla p_{N}^{*, n+\frac{1}{2}}\left(x_{i}, y_{j}\right)=\boldsymbol{f}^{n+\frac{1}{2}}\left(x_{i}, y_{j}\right), \quad j=1,2, \cdots, N-1, \\
& \boldsymbol{u}_{N}^{n+1}\left(x_{i}, \pm 1\right)=\mathbf{0} .
\end{aligned}
$$

In the above,

$$
p_{N}^{*, n+\frac{1}{2}}=p_{N}^{n-\frac{1}{2}}+\phi_{N}^{n-\frac{1}{2}}
$$


- Pressure splitting:

Find $\psi_{N}^{n+\frac{1}{2}} \in \mathbb{P}_{N}(\Omega)$, such that for each $j \in\{0,1, \cdots, N\}, \psi_{N}^{n+\frac{1}{2}}\left(x, y_{j}\right) \in \mathbb{P}_{N}(\Lambda)$ satisfies

$$
\begin{aligned}
& \left(\psi_{N}^{n+\frac{1}{2}}\left(x, y_{j}\right), q_{1, N}(x)\right)_{x, N, \Lambda}+\left(\partial_{x} \psi_{N}^{n+\frac{1}{2}}\left(x, y_{j}\right), \partial_{x} q_{1, N}(x)\right)_{x, N, \Lambda} \\
& =\left(-\frac{\nabla \cdot u_{N}^{n+1}\left(x, y_{j}\right)}{\Delta t}, q_{1, N}(x)\right)_{x, N, \Lambda}, \quad \forall q_{1, N}(x) \in \mathbb{P}_{N}(\Lambda) ;
\end{aligned}
$$

Find $\phi_{N}^{n+\frac{1}{2}} \in \mathbb{P}_{N}(\Omega)$, such that for each $i \in\{0,1, \cdots, N\}, \phi_{N}^{n+\frac{1}{2}}\left(x_{i}, y\right) \in \mathbb{P}_{N}(\Lambda)$ satisfies

$$
\begin{aligned}
& \left(\phi_{N}^{n+\frac{1}{2}}\left(x_{i}, y\right), q_{2, N}(y)\right)_{y, N, \Lambda}+\left(\partial_{y} \phi_{N}^{n+\frac{1}{2}}\left(x_{i}, y\right), \partial_{y} q_{2, N}(y)\right)_{y, N, \Lambda} \\
& =\left(\psi_{N}^{n+\frac{1}{2}}\left(x_{i}, y\right), q_{2, N}(y)\right)_{y, N, \Lambda}, \quad \forall q_{2, N}(y) \in \mathbb{P}_{N}(\Lambda)
\end{aligned}
$$

and

$$
p_{N}^{n+\frac{1}{2}}=p_{N}^{n-\frac{1}{2}}+\phi_{N}^{n+\frac{1}{2}}-\chi v \nabla \cdot\left(\frac{1}{2}\left(\boldsymbol{u}_{N}^{n+1}+\boldsymbol{u}_{N}^{n}\right)\right) .
$$

Remark 2.1. By integration by parts in the above weak formulation (2.19)-(2.20), we can derive the following equivalent formulation: fies

Find $\psi_{N}^{n+\frac{1}{2}} \in \mathbb{P}_{N}(\Omega)$, such that for each $y_{j}, j=0,1, \cdots, N, \psi_{N}^{n+\frac{1}{2}}\left(x, y_{j}\right) \in \mathbb{P}_{N}(\Lambda)$ satis-

$$
\begin{aligned}
& \psi_{N}^{n+\frac{1}{2}}\left(x_{i}, y_{j}\right)-\partial_{x x} \psi_{N}^{n+\frac{1}{2}}\left(x_{i}, y_{j}\right)=-\frac{\nabla \cdot \boldsymbol{u}_{N}^{n+1}\left(x_{i}, y_{j}\right)}{\Delta t}, \quad i=1,2, \cdots, N-1, \\
& \partial_{x} \psi_{N}^{n+\frac{1}{2}}\left(-1, y_{j}\right)=\omega_{0}\left(\psi_{N}^{n+\frac{1}{2}}\left(-1, y_{j}\right)-\partial_{x x} \psi_{N}^{n+\frac{1}{2}}\left(-1, y_{j}\right)+\frac{\nabla \cdot \boldsymbol{u}_{N}^{n+1}\left(-1, y_{j}\right)}{\Delta t}\right), \\
& \partial_{x} \psi_{N}^{n+\frac{1}{2}}\left(1, y_{j}\right)=-\omega_{N}\left(\psi_{N}^{n+\frac{1}{2}}\left(1, y_{j}\right)-\partial_{x x} \psi_{N}^{n+\frac{1}{2}}\left(1, y_{j}\right)+\frac{\nabla \cdot \boldsymbol{u}_{N}^{n+1}\left(1, y_{j}\right)}{\Delta t}\right) .
\end{aligned}
$$

Find $\phi_{N}^{n+\frac{1}{2}} \in \mathbb{P}_{N}(\Omega)$, such that for each $x_{i}, i=0,1, \cdots, N, \phi_{N}^{n+\frac{1}{2}}\left(x_{i}, y\right) \in \mathbb{P}_{N}(\Lambda)$ satisfies

$$
\begin{aligned}
& \phi_{N}^{n+\frac{1}{2}}\left(x_{i}, y_{j}\right)-\partial_{y y} \phi_{N}^{n+\frac{1}{2}}\left(x_{i}, y_{j}\right)=\psi_{N}^{n+\frac{1}{2}}\left(x_{i}, y_{j}\right), \quad j=1,2, \cdots, N-1, \\
& \partial_{y} \phi_{N}^{n+\frac{1}{2}}\left(x_{i},-1\right)=\omega_{0}\left(\phi_{N}^{n+\frac{1}{2}}\left(x_{i},-1\right)-\partial_{y y} \phi_{N}^{n+\frac{1}{2}}\left(x_{i},-1\right)-\psi_{N}^{n+\frac{1}{2}}\left(x_{i},-1\right)\right), \\
& \partial_{y} \phi_{N}^{n+\frac{1}{2}}\left(x_{i}, 1\right)=-\omega_{N}\left(\phi_{N}^{n+\frac{1}{2}}\left(x_{i}, 1\right)-\partial_{y y} \phi_{N}^{n+\frac{1}{2}}\left(x_{i}, 1\right)-\psi_{N}^{n+\frac{1}{2}}\left(x_{i}, 1\right)\right) .
\end{aligned}
$$

Note that in the above, $\psi_{N}^{n+1 / 2}$ (resp. $\phi_{N}^{n+1 / 2}$ ) satisfies the differential equation (2.11) (resp. (2.12)) exactly at all interior collocation points, while the homogeneous Neumann boundary conditions are satisfied only approximately with the error being the residual of the differential equation multiplied by the weight $\omega_{0}$ or $\omega_{N}$ which goes zero as $N$ tends to infinity. 


\section{Stability Analysis}

We shall prove that the scheme (2.16)-(2.21) is unconditionally stable by following a similar procedure as in [6].

We define the bilinear form $a_{N}(\cdot, \cdot)$ by:

$$
\begin{aligned}
a_{N}\left(p_{N}, q_{N}\right)= & \left(p_{N}, q_{N}\right)_{N, \Omega}+\left(\partial_{x} p_{N}, \partial_{x} q_{N}\right)_{N, \Omega}+\left(\partial_{y} p_{N}, \partial_{y} q_{N}\right)_{N, \Omega} \\
& +\left(\partial_{x y} p_{N}, \partial_{x y} q_{N}\right)_{N, \Omega}, \quad \forall p_{N}, q_{N} \in \mathbb{P}_{N}(\Omega) .
\end{aligned}
$$

Then it is easy to verify that $a_{N}(\cdot, \cdot)$ satisfies the following properties:

$$
a_{N}(\cdot, \cdot) \text { is symmetric, and }\left\|\nabla q_{N}\right\|_{N}^{2} \leq a_{N}\left(q_{N}, q_{N}\right), \quad \forall q_{N} \in \mathbb{P}_{N}(\Omega) .
$$

We also introduce the $A$-norm $\|\cdot\|_{A}$ and $A_{N}$-norm $\|\cdot\|_{A_{N}}$ defined by

$$
\|p\|_{A}:=a(p, p)^{\frac{1}{2}}, \quad\left\|p_{N}\right\|_{A_{N}}:=a_{N}\left(p_{N}, p_{N}\right)^{\frac{1}{2}} .
$$

By virtue of the definitions (2.7) and (3.1), it is readily seen that the two norms $\|\cdot\|_{A}$ and $\|\cdot\|_{A_{N}}$ are equivalent in $\mathbb{P}_{N}(\Omega)$.

We define the discrete operator $\delta$ by

$$
\delta p_{N}^{n+\frac{1}{2}}:=p_{N}^{n+\frac{1}{2}}-p_{N}^{n-\frac{1}{2}}, \quad \delta^{2} p_{N}^{n+\frac{1}{2}}:=p_{N}^{n+\frac{1}{2}}-2 p_{N}^{n-\frac{1}{2}}+p_{N}^{n-\frac{3}{2}} .
$$

We also use $\overline{\boldsymbol{u}}_{N}$ to denote the sequence whose generic term is $\overline{\boldsymbol{u}}_{N}^{n+\frac{1}{2}}:=\frac{1}{2}\left(\boldsymbol{u}_{N}^{n+1}+\boldsymbol{u}_{N}^{n}\right)$.

For any norm $\|\cdot\|_{E}$, we denote

$$
\|u\|_{l^{2}(0, T ; E)}^{2}=\Delta t \sum_{n=1}^{K}\left\|u\left(t^{n}, \cdot\right)\right\|_{E}^{2}, \quad\|u\|_{l^{\infty}(0, T ; E)}^{2}=\max _{0 \leq n \leq K}\left\|u\left(t^{n}, \cdot\right)\right\|_{E}^{2} .
$$

We start by proving the following lemma.

Lemma 3.1. The pressure splitting scheme (2.19)-(2.20) is equivalent to: Find $\phi_{N}^{n+\frac{1}{2}} \in \mathbb{P}_{N}(\Omega)$, such that

$$
a_{N}\left(\phi_{N}^{n+\frac{1}{2}}, q_{N}\right)=\left(-\frac{\nabla \cdot u_{N}^{n+1}}{\Delta t}, q_{N}\right)_{N, \Omega}, \quad \forall q_{N} \in \mathbb{P}_{N}(\Omega) .
$$

Proof. Note that for any $q_{2, N}(y) \in \mathbb{P}_{N}(\Lambda)$ both functions $\left(\phi_{N}^{n+\frac{1}{2}}(x, y), q_{2, N}(y)\right)_{y, N, \Lambda}+$ $\left(\partial_{y} \phi_{N}^{n+\frac{1}{2}}(x, y), \partial_{y} q_{2, N}(y)\right)_{y, N, \Lambda}$ and $\left(\psi_{N}^{n+\frac{1}{2}}(x, y), q_{2, N}(y)\right)_{y, N, \Lambda}$ are polynomials of degree not exceeding $N$ with respect to $x$, and they are equal at the $N+1$ Legendre-Gauss-Lobatto points according to equation (2.20). As a result, the two polynomials are identical, i.e.,

$$
\begin{aligned}
& \left(\phi_{N}^{n+\frac{1}{2}}(x, y), q_{2, N}(y)\right)_{y, N, \Lambda}+\left(\partial_{y} \phi_{N}^{n+\frac{1}{2}}(x, y), \partial_{y} q_{2, N}(y)\right)_{y, N, \Lambda} \\
& =\left(\psi_{N}^{n+\frac{1}{2}}(x, y), q_{2, N}(y)\right)_{y, N, \Lambda}, \forall q_{2, N}(y) \in \mathbb{P}_{N}(\Lambda), \forall x \in \Lambda .
\end{aligned}
$$


Differentiating both sides with respect to $x$ gives

$$
\begin{aligned}
& \left(\partial_{x} \phi_{N}^{n+\frac{1}{2}}\left(x_{i}, y\right), q_{2, N}(y)\right)_{y, N, \Lambda}+\left(\partial_{x y} \phi_{N}^{n+\frac{1}{2}}\left(x_{i}, y\right), \partial_{y} q_{2, N}(y)\right)_{y, N, \Lambda} \\
& =\left(\partial_{x} \psi_{N}^{n+\frac{1}{2}}\left(x_{i}, y\right), q_{2, N}(y)\right)_{y, N, \Lambda}, \forall q_{2, N}(y) \in \mathbb{P}_{N}(\Lambda), \quad \forall i=0,1, \cdots, N .
\end{aligned}
$$

For any $q_{1, N}(x) \in \mathbb{P}_{N}(\Lambda)$, multiplying both sides of equation (3.6) by $\partial_{x} q_{1, N}\left(x_{i}\right) \omega_{i}$ and summing up from $i=0,1, \cdots, N$, we obtain

$$
\begin{aligned}
& \left(\partial_{x} \phi_{N}^{n+\frac{1}{2}}(x, y), \partial_{x} q_{1, N}(x) q_{2, N}(y)\right)_{N, \Omega}+\left(\partial_{x y} \phi_{N}^{n+\frac{1}{2}}(x, y), \partial_{x} q_{1, N}(x) \partial_{y} q_{2, N}(y)\right)_{N, \Omega} \\
& =\left(\partial_{x} \psi_{N}^{n+\frac{1}{2}}(x, y), \partial_{x} q_{1, N}(x) q_{2, N}(y)\right)_{N, \Omega}, \forall q_{1, N}(x), q_{2, N}(y) \in \mathbb{P}_{N}(\Lambda) .
\end{aligned}
$$

Similarly, multiplying (2.20) by $q_{1, N}\left(x_{i}\right) \omega_{i}$ and summing up from $i=0,1, \cdots, N$, we get

$$
\begin{aligned}
& \left(\phi_{N}^{n+\frac{1}{2}}(x, y), q_{1, N}(x) q_{2, N}(y)\right)_{N, \Omega}+\left(\partial_{y} \phi_{N}^{n+\frac{1}{2}}(x, y), q_{1, N}(x) \partial_{y} q_{2, N}(y)\right)_{N, \Omega} \\
& =\left(\psi_{N}^{n+\frac{1}{2}}(x, y), q_{1, N}(x) q_{2, N}(y)\right)_{N, \Omega}, \forall q_{1, N}(x), q_{2, N}(y) \in \mathbb{P}_{N}(\Lambda) .
\end{aligned}
$$

Combining (3.8) and (3.7) yields

$$
\begin{gathered}
a_{N}\left(\phi_{N}^{n+\frac{1}{2}}, q_{1, N}(x) q_{2, N}(y)\right) \\
=\left(\psi_{N}^{n+\frac{1}{2}}(x, y), q_{1, N}(x) q_{2, N}(y)\right)_{N, \Omega}+\left(\partial_{x} \psi_{N}^{n+\frac{1}{2}}(x, y), \partial_{x} q_{1, N}(x) q_{2, N}(y)\right)_{N, \Omega}, \\
\forall q_{1, N}(x), q_{2, N}(y) \in \mathbb{P}_{N}(\Lambda) .
\end{gathered}
$$

Now we multiply (2.19) by $q_{2, N}\left(y_{j}\right) \omega_{j}$ and sum up over $j=0,1, \cdots, N$ to get

$$
\begin{aligned}
& \left(\psi_{N}^{n+\frac{1}{2}}(x, y), q_{1, N}(x) q_{2, N}(y)\right)_{N, \Omega}+\left(\partial_{x} \psi_{N}^{n+\frac{1}{2}}(x, y), \partial_{x} q_{1, N}(x) q_{2, N}(y)\right)_{N, \Omega} \\
& =\left(-\frac{\nabla \cdot \boldsymbol{u}_{N}^{n+1}(x, y)}{\Delta t}, q_{1, N}(x) q_{2, N}(y)\right)_{N, \Omega}, \quad \forall q_{1, N}(x), q_{2, N}(y) \in \mathbb{P}_{N}(\Lambda) .
\end{aligned}
$$

By comparing (3.10) with (3.9), we obtain

$$
\begin{aligned}
& a_{N}\left(\phi_{N}^{n+\frac{1}{2}}, q_{1, N}(x) q_{2, N}(y)\right) \\
& =\left(-\frac{\nabla \cdot \boldsymbol{u}_{N}^{n+1}}{\Delta t}, q_{1, N}(x) q_{2, N}(y)\right)_{N, \Omega}, \quad \forall q_{1, N}(x), q_{2, N}(y) \in \mathbb{P}_{N}(\Lambda),
\end{aligned}
$$

which implies (3.4).

We are now in positive to prove the stability of the scheme. We start with the standard case, i.e., $\chi=0$. Without loss of generality, we assume $\boldsymbol{f}=\mathbf{0}$. 
Theorem 3.1. The solution to (2.16)-(2.21) with $f=0$ and $\chi=0$ satisfies the following inequality:

$$
\begin{aligned}
& \left\|\boldsymbol{u}_{N}\right\|_{l^{\infty}\left(0, T ; L^{2}\right)}^{2}+\frac{v}{2}\left\|\nabla \boldsymbol{u}_{N}\right\|_{l^{2}\left(0, T ; L^{2}\right)}^{2}+\Delta t\left\|p_{N}\right\|_{l^{2}\left(-\frac{\Delta t}{2}, T-\frac{\Delta t}{2} ; A\right)}^{2}+\frac{\Delta t v^{2}}{4}\left\|\partial_{x y} \boldsymbol{u}_{N}^{n+1}\right\|_{l^{2}\left(0, T ; L^{2}\right)}^{2} \\
& \leq c\left(\left\|\boldsymbol{u}_{0}\right\|_{L^{2}}^{2}+\frac{v}{2}\left\|\nabla \boldsymbol{u}_{0}\right\|_{L^{2}}^{2}+\Delta t\left\|p_{0}\right\|_{A}^{2}+\frac{\Delta t v^{2}}{4}\left\|\partial_{x y} \boldsymbol{u}_{0}\right\|_{L^{2}}^{2}\right)
\end{aligned}
$$

where $c$ is a constant independent of discretization parameters.

Proof. Subtracting equation (2.17) from (2.16), we obtain the following expression for the half-step discrete velocity:

$$
\boldsymbol{u}_{N}^{n+\frac{1}{2}}\left(\boldsymbol{x}_{i j}\right)=\frac{\boldsymbol{u}_{N}^{n+1}\left(\boldsymbol{x}_{i j}\right)+\boldsymbol{u}_{N}^{n}\left(\boldsymbol{x}_{i j}\right)}{2}-\frac{\Delta t v}{4} \partial_{y y}\left(\boldsymbol{u}_{N}^{n+1}\left(\boldsymbol{x}_{i j}\right)-\boldsymbol{u}_{N}^{n}\left(\boldsymbol{x}_{i j}\right)\right), \quad \forall \boldsymbol{x}_{i j} \in \Sigma_{I} .
$$

On the other hand, adding (2.17) to (2.16) with $f=0$, we find that

$\frac{\boldsymbol{u}_{N}^{n+1}\left(\boldsymbol{x}_{i j}\right)-\boldsymbol{u}_{N}^{n}\left(\boldsymbol{x}_{i j}\right)}{\Delta t}-v \partial_{x x} \boldsymbol{u}_{N}^{n+\frac{1}{2}}\left(\boldsymbol{x}_{i j}\right)-v \partial_{y y} \overline{\boldsymbol{u}}_{N}^{n+\frac{1}{2}}\left(\boldsymbol{x}_{i j}\right)+\nabla p_{N}^{*, n+\frac{1}{2}}\left(\boldsymbol{x}_{i j}\right)=\mathbf{0}, \quad \forall \boldsymbol{x}_{i j} \in \Sigma_{I}$.

Note that the functions on both sides of (3.11) are polynomials of degree $\leq N$ with respect to $x$, which are equal at $N-1$ interior nodes according to (3.11) and also at two end points due to the fact that

$$
\boldsymbol{u}_{N}^{n+\frac{1}{2}}\left( \pm 1, y_{j}\right)=\boldsymbol{u}_{N}^{n}\left( \pm 1, y_{j}\right)=\mathbf{0}, \quad \forall n=0,1, \cdots, K .
$$

Thus from (3.11), we obtain

$$
\begin{gathered}
\boldsymbol{u}_{N}^{n+\frac{1}{2}}\left(x, y_{j}\right)=\frac{\boldsymbol{u}_{N}^{n+1}\left(x, y_{j}\right)+\boldsymbol{u}_{N}^{n}\left(x, y_{j}\right)}{2}-\frac{\Delta t v}{4} \partial_{y y}\left(\boldsymbol{u}_{N}^{n+1}\left(x, y_{j}\right)-\boldsymbol{u}_{N}^{n}\left(x, y_{j}\right)\right), \\
\forall x \in \Lambda, j=1, \cdots, N-1 .
\end{gathered}
$$

Taking two derivatives w.r.t. $x$, we get

$$
\partial_{x x} \boldsymbol{u}_{N}^{n+\frac{1}{2}}\left(\boldsymbol{x}_{i j}\right)=\partial_{x x} \overline{\boldsymbol{u}}_{N}^{n+\frac{1}{2}}\left(\boldsymbol{x}_{i j}\right)-\frac{\Delta t v}{4} \partial_{x x y y}\left(\boldsymbol{u}_{N}^{n+1}\left(\boldsymbol{x}_{i j}\right)-\boldsymbol{u}_{N}^{n}\left(\boldsymbol{x}_{i j}\right)\right), \quad \forall \boldsymbol{x}_{i j} \in \Sigma_{I} .
$$

Plugging (3.13) into (3.12) leads to

$$
\begin{aligned}
& \frac{\boldsymbol{u}_{N}^{n+1}\left(\boldsymbol{x}_{i j}\right)-\boldsymbol{u}_{N}^{n}\left(\boldsymbol{x}_{i j}\right)}{\Delta t}-v \Delta \overline{\boldsymbol{u}}_{N}^{n+\frac{1}{2}}\left(\boldsymbol{x}_{i j}\right)+\nabla p_{N}^{*, n+\frac{1}{2}}\left(\boldsymbol{x}_{i j}\right)+\frac{\Delta t v^{2}}{4} \partial_{x x y y}\left(\boldsymbol{u}_{N}^{n+1}\left(\boldsymbol{x}_{i j}\right)-\boldsymbol{u}_{N}^{n}\left(\boldsymbol{x}_{i j}\right)\right) \\
& =\mathbf{0}, \quad \forall \boldsymbol{x}_{i j} \in \Sigma_{I} .
\end{aligned}
$$


We multiply (3.14) by $\boldsymbol{u}_{N}^{n+1}\left(\boldsymbol{x}_{i j}\right) \omega_{i} \omega_{j}$, and sum up over $i, j$ from 1 to $N-1$ to yield

$$
\begin{aligned}
& \left(\frac{\boldsymbol{u}_{N}^{n+1}-\boldsymbol{u}_{N}^{n}}{\Delta t}, \boldsymbol{u}_{N}^{n+1}\right)_{N}-v\left(\Delta \overline{\boldsymbol{u}}_{N}^{n+\frac{1}{2}}, \boldsymbol{u}_{N}^{n+1}\right)_{N}+\left(\nabla p_{N}^{*, n+\frac{1}{2}}, \boldsymbol{u}_{N}^{n+1}\right)_{N} \\
& +\frac{\Delta t v^{2}}{4}\left(\partial_{x x y y}\left(\boldsymbol{u}_{N}^{n+1}-\boldsymbol{u}_{N}^{n}\right), \boldsymbol{u}_{N}^{n+1}\right)_{N}=\mathbf{0} .
\end{aligned}
$$

Integrating by parts in the second term and the last term, thanks to the boundary conditions and the exactness of the one-dimensional Legendre-Gauss-Lobatto quadrature, and using the identity $2(a-b, a)=\|a\|^{2}+\|a-b\|^{2}-\|b\|^{2}$, we find

$$
\begin{aligned}
& \left\|\boldsymbol{u}_{N}^{n+1}\right\|_{N}^{2}+\left\|\boldsymbol{u}_{N}^{n+1}-\boldsymbol{u}_{N}^{n}\right\|_{N}^{2}+\frac{\Delta t v}{2}\left(\left\|\nabla \boldsymbol{u}_{N}^{n+1}\right\|_{N}^{2}+\left\|\nabla\left(\boldsymbol{u}_{N}^{n+1}+\boldsymbol{u}_{N}^{n}\right)\right\|_{N}^{2}\right) \\
& \quad+2 \Delta t\left(\nabla p_{N}^{*, n+\frac{1}{2}}, \boldsymbol{u}_{N}^{n+1}\right)_{N}+\frac{\Delta t^{2} v^{2}}{4}\left\|\partial_{x y} \boldsymbol{u}_{N}^{n+1}\right\|_{N}^{2}+\frac{\Delta t^{2} v^{2}}{4}\left\|\partial_{x y}\left(\boldsymbol{u}_{N}^{n+1}-\boldsymbol{u}_{N}^{n}\right)\right\|_{N}^{2} \\
& =\left\|\boldsymbol{u}_{N}^{n}\right\|_{N}^{2}+\frac{\Delta t v}{2}\left\|\nabla \boldsymbol{u}_{N}^{n}\right\|_{N}^{2}+\frac{\Delta t^{2} v^{2}}{4}\left\|\partial_{x y} \boldsymbol{u}_{N}^{n}\right\|_{N}^{2} .
\end{aligned}
$$

On the other hand, from (2.21) with $\chi=0$, we have

$$
\phi_{N}^{n+\frac{1}{2}}=p_{N}^{n+\frac{1}{2}}-p_{N}^{n-\frac{1}{2}} \text { and } p_{N}^{*, n+\frac{1}{2}}=2 p_{N}^{n-\frac{1}{2}}-p_{N}^{n-\frac{3}{2}} .
$$

In virtue of (3.4), the function $p_{N}^{n+\frac{1}{2}}-p_{N}^{n-\frac{1}{2}} \in \mathbb{P}_{N}(\Omega)$ satisfies: for $n \geq 0$,

$$
a_{N}\left(p_{N}^{n+\frac{1}{2}}-p_{N}^{n-\frac{1}{2}}, q_{N}\right)=-\frac{1}{\Delta t}\left(\nabla \cdot \boldsymbol{u}_{N}^{n+1}, q_{N}\right)_{N}, \quad \forall q_{N} \in \mathbb{P}_{N}(\Omega)
$$

Taking $q_{N}=2 \Delta t^{2} p_{N}^{*, n+\frac{1}{2}}:=2 \Delta t^{2}\left(2 p_{N}^{n-\frac{1}{2}}-p_{N}^{n-\frac{3}{2}}\right)$ in the above, and using the fact that $a_{N}(\cdot, \cdot)$ is symmetric, we have

$$
\begin{aligned}
& -2 \Delta t\left(\nabla \cdot \boldsymbol{u}_{N}^{n+1}, p_{N}^{*, n+\frac{1}{2}}\right)_{N} \\
& =2 \Delta t^{2} a_{N}\left(\delta p_{N}^{n+\frac{1}{2}}, 2 p_{N}^{n-\frac{1}{2}}-p_{N}^{n-\frac{3}{2}}\right) \\
& =2 \Delta t^{2} a_{N}\left(\delta p_{N}^{n+\frac{1}{2}}, p_{N}^{n+\frac{1}{2}}\right)+2 \Delta t^{2} a_{N}\left(\delta p_{N}^{n+\frac{1}{2}},-\delta p_{N}^{n+\frac{1}{2}}+\delta p_{N}^{n-\frac{1}{2}}\right) \\
& =\Delta t^{2}\left(\left\|p_{N}^{n+\frac{1}{2}}\right\|_{A_{N}}^{2}-\left\|p_{N}^{n-\frac{1}{2}}\right\|_{A_{N}}^{2}+\left\|\delta p_{N}^{n-\frac{1}{2}}\right\|_{A_{N}}^{2}-\left\|\delta^{2} p_{N}^{n+\frac{1}{2}}\right\|_{A_{N}}^{2}\right) .
\end{aligned}
$$

Next we seek to bound the term $\left\|\delta^{2} p_{N}^{n+\frac{1}{2}}\right\|_{A_{N}}^{2}$. Applying the time increment operator $\delta$ to (3.18) and taking $q_{N}=\Delta t \delta^{2} p_{N}^{n+\frac{1}{2}}$ in the resulting equation, we obtain

$$
\begin{aligned}
\Delta t\left\|\delta^{2} p_{N}^{n+\frac{1}{2}}\right\|_{A_{N}}^{2} & =-\left(\nabla \cdot\left(\boldsymbol{u}_{N}^{n+1}-\boldsymbol{u}_{N}^{n}\right), \delta^{2} p_{N}^{n+\frac{1}{2}}\right)_{N} \\
& =\left(\boldsymbol{u}_{N}^{n+1}-\boldsymbol{u}_{N}^{n}, \nabla \delta^{2} p_{N}^{n+\frac{1}{2}}\right)_{N} \leq\left\|\boldsymbol{u}_{N}^{n+1}-\boldsymbol{u}_{N}^{n}\right\|_{N}\left\|\nabla \delta^{2} p_{N}^{n+\frac{1}{2}}\right\|_{N} .
\end{aligned}
$$


Then by using the coercivity (3.2), we obtain

$$
\Delta t\left\|\nabla \delta^{2} p_{N}^{n+\frac{1}{2}}\right\|_{N}^{2} \leq\left\|\boldsymbol{u}_{N}^{n+1}-\boldsymbol{u}_{N}^{n}\right\|_{N}\left\|\nabla \delta^{2} p_{N}^{n+\frac{1}{2}}\right\|_{A_{N}},
$$

which results in

$$
\Delta t^{2}\left\|\delta^{2} p_{N}^{n+\frac{1}{2}}\right\|_{A_{N}}^{2} \leq\left\|\boldsymbol{u}_{N}^{n+1}-\boldsymbol{u}_{N}^{n}\right\|_{N}^{2} .
$$

Inserting this bound into (3.19) gives

$$
\begin{aligned}
& \Delta t^{2}\left(\left\|p_{N}^{n+\frac{1}{2}}\right\|_{A_{N}}^{2}+\left\|\delta p_{N}^{n-\frac{1}{2}}\right\|_{A_{N}}^{2}-\left\|p_{N}^{n-\frac{1}{2}}\right\|_{A_{N}}^{2}\right) \\
& \leq-2 \Delta t\left(\nabla \cdot \boldsymbol{u}_{N}^{n+1}, p_{N}^{*, n+\frac{1}{2}}\right)_{N}+\left\|\boldsymbol{u}_{N}^{n+1}-\boldsymbol{u}_{N}^{n}\right\|_{N}^{2} .
\end{aligned}
$$

Then, combining the above estimate with (3.16), noticing that

$$
\left(\nabla \cdot \boldsymbol{u}_{N}^{n+1}, p_{N}^{*, n+\frac{1}{2}}\right)_{N}=-\left(\nabla p_{N}^{*, n+\frac{1}{2}}, \boldsymbol{u}_{N}^{n+1}\right)_{N},
$$

and dropping some unnecessary positive terms, we arrive at

$$
\begin{aligned}
& \left\|\boldsymbol{u}_{N}^{n+1}\right\|_{N}^{2}+\frac{\Delta t v}{2}\left\|\nabla \boldsymbol{u}_{N}^{n+1}\right\|_{N}^{2}+\Delta t^{2}\left\|p_{N}^{n+\frac{1}{2}}\right\|_{A_{N}}^{2}+\frac{\Delta t^{2} v^{2}}{4}\left\|\partial_{x y} \boldsymbol{u}_{N}^{n+1}\right\|_{N}^{2} \\
& \leq\left\|\boldsymbol{u}_{N}^{n}\right\|_{N}^{2}+\frac{\Delta t v}{2}\left\|\nabla \boldsymbol{u}_{N}^{n}\right\|_{N}^{2}+\Delta t^{2}\left\|p_{N}^{n-\frac{1}{2}}\right\|_{A_{N}}^{2}+\frac{\Delta t^{2} v^{2}}{4}\left\|\partial_{x y} \boldsymbol{u}_{N}^{n}\right\|_{N}^{2} .
\end{aligned}
$$

Finally, the desired result is obtained by summing up the above estimate and using the norm equivalences between $\|\cdot\|_{A}$ and $\|\cdot\|_{A_{N}}$, and between $\|\cdot\|_{N}$ and $\|\cdot\|_{L^{2}}$.

Next, we establish the stability for the scheme (2.18)-(2.21) in the rotational form, i.e., with $\chi \neq 0$.

Theorem 3.2. The solution to (2.16)-(2.21) with $f=0$ and $0<\chi \leq 1$ satisfies the following inequality:

$$
\begin{aligned}
& \left\|\delta \boldsymbol{u}_{N}^{n+1}\right\|_{l^{\infty}\left(0, T ; L^{2}\right)}^{2}+\frac{v}{2}\left\|\nabla \times \delta \boldsymbol{u}_{N}^{n+1}\right\|_{l^{2}\left(0, T ; L^{2}\right)}^{2}+\frac{v}{2}(1-\chi)\left\|\nabla \cdot \delta \boldsymbol{u}_{N}^{n+1}\right\|_{L^{2}\left(0, T ; L^{2}\right)}^{2} \\
& \quad+\frac{\Delta t v^{2}}{4}\left\|\partial_{x y} \delta \boldsymbol{u}_{N}^{n+1}\right\|_{L^{2}\left(0, T ; L^{2}\right)}^{2}+\Delta t\left\|\phi_{N}^{n+\frac{1}{2}}\right\|_{l^{2}\left(-\frac{\Delta t}{2}, T-\frac{\Delta t}{2} ; A\right)}^{2}+\chi v\left\|\nabla \cdot \boldsymbol{u}_{N}^{n+1}\right\|_{l^{2}\left(0, T ; L^{2}\right)}^{2} \\
& \leq c\left(\left\|\delta \boldsymbol{u}_{N}^{1}\right\|_{L^{2}}^{2}+\frac{v}{2}\left\|\nabla \times \delta \boldsymbol{u}_{N}^{1}\right\|_{L^{2}}^{2}+\frac{v}{2}(1-\chi)\left\|\nabla \cdot \delta \boldsymbol{u}_{N}^{1}\right\|_{L^{2}}^{2}\right. \\
& \left.\quad+\frac{\Delta t v^{2}}{4}\left\|\partial_{x y} \delta \boldsymbol{u}_{N}^{1}\right\|_{L^{2}}^{2}+\Delta t\left\|\phi_{N}^{\frac{1}{2}}\right\|_{A}^{2}+\chi v\left\|\nabla \cdot \boldsymbol{u}_{N}^{1}\right\|_{L^{2}}^{2}\right),
\end{aligned}
$$

where $c$ is a constant independent of discretization parameters. 
Proof. Applying the time increment operator $\delta$ to (3.14) and taking the discrete inner product with $\delta \boldsymbol{u}_{N}^{n+1}$, we obtain

$$
\begin{aligned}
& \left(\frac{\delta \boldsymbol{u}_{N}^{n+1}-\delta \boldsymbol{u}_{N}^{n}}{\Delta t}, \delta \boldsymbol{u}_{N}^{n+1}\right)_{N}-v\left(\Delta \delta \overline{\boldsymbol{u}}_{N}^{n+\frac{1}{2}}, \delta \boldsymbol{u}_{N}^{n+1}\right)_{N}+\left(\nabla \delta p_{N}^{*, n+\frac{1}{2}}, \delta \boldsymbol{u}_{N}^{n+1}\right)_{N} \\
& +\frac{\Delta t v^{2}}{4}\left(\partial_{x x y y} \delta\left(\boldsymbol{u}_{N}^{n+1}-\boldsymbol{u}_{N}^{n}\right), \delta \boldsymbol{u}_{N}^{n+1}\right)_{N}=\mathbf{0}
\end{aligned}
$$

As in the proof of previous theorem, we integrate by parts in the second term and the last term to obtain

$$
\begin{aligned}
& \left\|\delta \boldsymbol{u}_{N}^{n+1}\right\|_{N}^{2}+\left\|\delta^{2} \boldsymbol{u}_{N}^{n+1}\right\|_{N}^{2}+\frac{\Delta t v}{2}\left(\left\|\nabla \delta \boldsymbol{u}_{N}^{n+1}\right\|_{N}^{2}+\left\|\nabla \delta\left(\boldsymbol{u}_{N}^{n+1}+\boldsymbol{u}_{N}^{n}\right)\right\|_{N}^{2}\right) \\
& +2 \Delta t\left(\nabla \delta p_{N}^{*, n+\frac{1}{2}}, \delta \boldsymbol{u}_{N}^{n+1}\right)_{N}+\frac{\Delta t^{2} v^{2}}{4}\left\|\partial_{x y} \delta \boldsymbol{u}_{N}^{n+1}\right\|_{N}^{2}+\frac{\Delta t^{2} v^{2}}{4}\left\|\partial_{x y} \delta\left(\boldsymbol{u}_{N}^{n+1}-\boldsymbol{u}_{N}^{n}\right)\right\|_{N}^{2} \\
& =\left\|\delta \boldsymbol{u}_{N}^{n}\right\|_{N}^{2}+\frac{\Delta t v}{2}\left\|\nabla \delta \boldsymbol{u}_{N}^{n}\right\|_{N}^{2}+\frac{\Delta t^{2} v^{2}}{4}\left\|\partial_{x y} \delta \boldsymbol{u}_{N}^{n}\right\|_{N}^{2}
\end{aligned}
$$

Rewrite (2.21) as

$$
\phi_{N}^{n+\frac{1}{2}}=p_{N}^{n+\frac{1}{2}}-p_{N}^{n-\frac{1}{2}}+\chi v \nabla \cdot\left(\frac{1}{2}\left(\boldsymbol{u}_{N}^{n+1}+\boldsymbol{u}_{N}^{n}\right)\right)=\delta p_{N}^{n+\frac{1}{2}}+\frac{1}{2} \chi v \nabla \cdot\left(\boldsymbol{u}_{N}^{n+1}+\boldsymbol{u}_{N}^{n}\right) .
$$

Applying the time increment operator $\delta$ to (3.4) and taking $q_{N}=2 \Delta t^{2} \phi_{N}^{n+\frac{1}{2}}$, using the above relation, we obtain

$$
\begin{aligned}
& \Delta t^{2}\left(\left\|\phi_{N}^{n+\frac{1}{2}}\right\|_{A_{N}}^{2}+\left\|\delta \phi_{N}^{n+\frac{1}{2}}\right\|_{A_{N}}^{2}-\left\|\phi_{N}^{n-\frac{1}{2}}\right\|_{A_{N}}^{2}\right) \\
& =-2 \Delta t\left(\nabla \cdot \delta \boldsymbol{u}_{N}^{n+1}, \phi_{N}^{n+\frac{1}{2}}\right)_{N} \\
& =-2 \Delta t\left(\nabla \cdot \delta \boldsymbol{u}_{N}^{n+1}, \delta p_{N}^{n+\frac{1}{2}}+\frac{1}{2} \chi v \nabla \cdot\left(\boldsymbol{u}_{N}^{n+1}+\boldsymbol{u}_{N}^{n}\right)\right)_{N},
\end{aligned}
$$

from which we derive

$$
\begin{aligned}
& \Delta t^{2}\left(\left\|\phi_{N}^{n+\frac{1}{2}}\right\|_{A_{N}}^{2}+\left\|\delta \phi_{N}^{n+\frac{1}{2}}\right\|_{A_{N}}^{2}\right)+\chi v \Delta t\left\|\nabla \cdot \boldsymbol{u}_{N}^{n+1}\right\|_{N}^{2} \\
& =\Delta t^{2}\left\|\phi_{N}^{n-\frac{1}{2}}\right\|_{A_{N}}^{2}+\chi v \Delta t\left\|\nabla \cdot \boldsymbol{u}_{N}^{n}\right\|_{N}^{2}+2 \Delta t\left(\delta \boldsymbol{u}_{N}^{n+1}, \nabla \delta p_{N}^{n+\frac{1}{2}}\right)_{N} .
\end{aligned}
$$

Applying the time increment operator $\delta$ to (3.18) and taking $q_{N}=-2 \Delta t^{2} \delta^{2} \phi_{N}^{n+\frac{1}{2}}$, we get

$$
-\Delta t^{2}\left(\left\|\delta \phi_{N}^{n+\frac{1}{2}}\right\|_{A_{N}}^{2}+\left\|\delta^{2} \phi_{N}^{n+\frac{1}{2}}\right\|_{A_{N}}^{2}-\left\|\delta \phi_{N}^{n-\frac{1}{2}}\right\|_{A_{N}}^{2}\right)=-2 \Delta t\left(\delta u_{N}^{n+1}, \nabla \delta^{2} \phi_{N}^{n+\frac{1}{2}}\right)_{N} .
$$


Summing up (3.21), (3.23) and (3.24), and using the following identities

$$
\begin{aligned}
& \delta p_{N}^{*, n+\frac{1}{2}}+\delta^{2} \phi_{N}^{n+\frac{1}{2}}-\delta p_{N}^{n+\frac{1}{2}}=\delta \phi_{N}^{n-\frac{1}{2}}+\delta p_{N}^{n-\frac{1}{2}}+\delta^{2} \phi_{N}^{n+\frac{1}{2}}-\delta p_{N}^{n+\frac{1}{2}} \\
& =\delta \phi_{N}^{n+\frac{1}{2}}-\delta^{2} p_{N}^{n+\frac{1}{2}}=\frac{1}{2} \chi v \nabla \cdot \delta\left(\boldsymbol{u}_{N}^{n+1}+\boldsymbol{u}_{N}^{n}\right) \\
& 2 \Delta t\left(\frac{1}{2} \chi v \nabla \cdot \delta\left(\boldsymbol{u}_{N}^{n+1}+\boldsymbol{u}_{N}^{n}\right), \nabla \cdot \delta \boldsymbol{u}_{N}^{n+1}\right)_{N} \\
& =\frac{1}{2} \chi v \Delta t\left(\left\|\nabla \cdot \delta \boldsymbol{u}_{N}^{n+1}\right\|_{N}^{2}+\left\|\nabla \cdot \delta\left(\boldsymbol{u}_{N}^{n+1}+\boldsymbol{u}_{N}^{n}\right)\right\|_{N}^{2}-\left\|\nabla \cdot \delta \boldsymbol{u}_{N}^{n}\right\|_{N}^{2}\right)
\end{aligned}
$$

and

$$
\|\nabla u\|_{L^{2}(\Omega)}^{2}=\|\nabla \times u\|_{L^{2}(\Omega)}^{2}+\|\nabla \cdot u\|_{L^{2}(\Omega)}^{2} \quad \forall u \in H_{0}^{1}(\Omega)^{2},
$$

we deduce that

$$
\begin{aligned}
\| & \delta \boldsymbol{u}_{N}^{n+1}\left\|_{N}^{2}+\right\| \delta^{2} \boldsymbol{u}_{N}^{n+1}\left\|_{N}^{2}+\frac{\Delta t v}{2}\right\| \nabla \times \delta \boldsymbol{u}_{N}^{n+1}\left\|_{N}^{2}+\frac{\Delta t v}{2}(1-\chi)\right\| \nabla \cdot \delta \boldsymbol{u}_{N}^{n+1} \|_{N}^{2} \\
& +\frac{\Delta t v}{2}\left\|\nabla \times \delta\left(\boldsymbol{u}_{N}^{n+1}+\boldsymbol{u}_{N}^{n}\right)\right\|_{N}^{2}+\frac{\Delta t v}{2}(1-\chi)\left\|\nabla \cdot \delta\left(\boldsymbol{u}_{N}^{n+1}+\boldsymbol{u}_{N}^{n}\right)\right\|_{N}^{2} \\
& +\frac{\Delta t^{2} v^{2}}{4}\left\|\partial_{x y} \delta \boldsymbol{u}_{N}^{n+1}\right\|_{N}^{2}+\frac{\Delta t^{2} v^{2}}{4}\left\|\partial_{x y} \delta\left(\boldsymbol{u}_{N}^{n+1}-\boldsymbol{u}_{N}^{n}\right)\right\|_{N}^{2} \\
& +\Delta t^{2}\left\|\phi_{N}^{n+\frac{1}{2}}\right\|_{A_{N}}^{2}+\chi v \Delta t\left\|\nabla \cdot \boldsymbol{u}_{N}^{n+1}\right\|_{N}^{2}+\Delta t^{2}\left\|\delta \phi_{N}^{n-\frac{1}{2}}\right\|_{A_{N}}^{2} \\
= & \left\|\delta \boldsymbol{u}_{N}^{n}\right\|_{N}^{2}+\frac{\Delta t v}{2}\left\|\nabla \times \delta \boldsymbol{u}_{N}^{n}\right\|_{N}^{2}+\frac{\Delta t v}{2}(1-\chi)\left\|\nabla \cdot \delta \boldsymbol{u}_{N}^{n}\right\|_{N}^{2} \\
& +\frac{\Delta t^{2} v^{2}}{4}\left\|\partial_{x y} \delta \boldsymbol{u}_{N}^{n}\right\|_{N}^{2}+\Delta t^{2}\left\|\phi_{N}^{n-\frac{1}{2}}\right\|_{A_{N}}^{2}+\chi v \Delta t\left\|\nabla \cdot \boldsymbol{u}_{N}^{n}\right\|_{N}^{2}+\Delta t^{2}\left\|\delta^{2} \phi_{N}^{n+\frac{1}{2}}\right\|_{A_{N}}^{2}
\end{aligned}
$$

It remains to estimate the last term. This can be done by applying the time increment operator $\delta^{2}$ to (3.4) and taking $q_{N}=\Delta t \delta^{2} \phi_{N}^{n+1}$ to obtain

$$
\Delta t\left\|\delta^{2} \phi_{N}^{n+\frac{1}{2}}\right\|_{A_{N}} \leq\left\|\delta^{2} \boldsymbol{u}_{N}^{n+1}\right\|_{N}
$$

Finally, the desired result is obtained by summing up in $n$ and using the norm equivalences between $\|\cdot\|_{A}$ and $\|\cdot\|_{A_{N}}$, and between $\|\cdot\|_{N}$ and $\|\cdot\|_{L^{2}}$.

\section{Navier-Stokes Equations with Variable Viscosity}

The main advantage of this new direction splitting method for solving the incompressible Navier-Stokes equations is that the scheme consists of solving a sequence of onedimensional elliptic problems, which can be solved in a very simple and efficient way. In this section, we generalize the scheme to treat the case with variable viscosity. 
We consider the following Navier-Stokes equations with variable viscosity:

$$
\begin{cases}\frac{\partial \boldsymbol{u}}{\partial t}-\nabla \cdot(v(x, y, t) \nabla \boldsymbol{u})+\boldsymbol{u} \cdot \nabla \boldsymbol{u}+\nabla p=\boldsymbol{f}, & \text { in } \Omega \times(0, T], \\ \nabla \cdot \boldsymbol{u}=0, & \text { in } \Omega \times[0, T], \\ \left.\boldsymbol{u}\right|_{\partial \Omega}=\mathbf{0}, & \text { in }[0, T], \\ \left.\boldsymbol{u}\right|_{t=0}=\boldsymbol{u}_{0}, & \text { in } \Omega,\end{cases}
$$

where $v(x, y, t)>0$ is the viscosity which can vary in time and in space. In real applications, the viscosity is usually determined by some other relations. While the above problem by itself does not model any realistic situation, one often needs to solve such a system as part of the solution process in multi-phase incompressible flows, Boussinesq flows, or the Navier-Stokes equations under a domain mapping. Therefore, it is very beneficial to have an efficient numerical method for (4.1).

We propose the following direction splitting scheme for solving (4.1). For the sake of simplicity, we omit the treatment of the nonlinear term below.

Setting $\boldsymbol{u}^{0}=\left.\boldsymbol{u}\right|_{t=0}, p^{-\frac{1}{2}}=\left.p\right|_{t=0}$ and $\phi^{-\frac{1}{2}}=0$. For $n \geq 0$, we look for $\left(\boldsymbol{u}^{n+\frac{1}{2}}, \boldsymbol{u}^{n+1}, p^{n+\frac{1}{2}}\right)$ as follows:

- Velocity splitting:

$$
\begin{aligned}
& \frac{\boldsymbol{u}^{n+\frac{1}{2}}-\boldsymbol{u}^{n}}{\frac{1}{2} \Delta t}-\partial_{x}\left(v^{n+\frac{1}{2}}(x, y) \partial_{x} \boldsymbol{u}^{n+\frac{1}{2}}\right)-\partial_{y}\left(v^{n+\frac{1}{2}}(x, y) \partial_{y} \boldsymbol{u}^{n}\right)+\nabla p^{*, n+\frac{1}{2}} \\
& =\boldsymbol{f}^{n+\frac{1}{2}},\left.\boldsymbol{u}^{n+\frac{1}{2}}\right|_{x= \pm 1}=\mathbf{0} \\
& \frac{\boldsymbol{u}^{n+1}-\boldsymbol{u}^{n+\frac{1}{2}}}{\frac{1}{2} \Delta t}-\partial_{x}\left(v^{n+\frac{1}{2}}(x, y) \partial_{x} \boldsymbol{u}^{n+\frac{1}{2}}\right)-\partial_{y}\left(v^{n+\frac{1}{2}}(x, y) \partial_{y} \boldsymbol{u}^{n+1}\right)+\nabla p^{*, n+\frac{1}{2}} \\
& =\boldsymbol{f}^{n+\frac{1}{2}},\left.\boldsymbol{u}^{n+1}\right|_{y= \pm 1}=\mathbf{0}
\end{aligned}
$$

where $v^{n+\frac{1}{2}}:=v\left(x, y, t^{n+\frac{1}{2}}\right)$.

- Pressure splitting:

$$
\begin{array}{ll}
\psi^{n+\frac{1}{2}}-\partial_{x x} \psi^{n+\frac{1}{2}}=-\frac{\nabla \cdot \boldsymbol{u}^{n+1}}{\Delta t} ; & \left.\partial_{x} \psi^{n+\frac{1}{2}}\right|_{x= \pm 1}=0 \\
\phi^{n+\frac{1}{2}}-\partial_{y y} \phi^{n+\frac{1}{2}}=\psi^{n+\frac{1}{2}} ; & \left.\partial_{y} \phi^{n+\frac{1}{2}}\right|_{y= \pm 1}=0
\end{array}
$$

and

$$
p^{n+\frac{1}{2}}=p^{n-\frac{1}{2}}+\phi^{n+\frac{1}{2}}-\chi v \nabla \cdot\left(\frac{1}{2}\left(\boldsymbol{u}^{n+1}+\boldsymbol{u}^{n}\right)\right) .
$$


It is easy to see that the above scheme has the same order of accuracy as the scheme (2.8)(2.13). However, it appears not easy to prove its stability directly. Therefore, we shall prove an intermediate result. Namely, we replace the velocity splitting step (4.2) by

$$
\begin{aligned}
& \frac{\boldsymbol{u}^{n+1}-\boldsymbol{u}^{n}}{\Delta t}-\frac{1}{2} \nabla \cdot\left(v^{n+\frac{1}{2}}(x, y) \nabla\left(\boldsymbol{u}^{n+1}+\boldsymbol{u}^{n}\right)\right)+\nabla p^{*, n+\frac{1}{2}}=\mathbf{0}, \\
& \left.\boldsymbol{u}^{n+1}\right|_{\partial \Omega}=\mathbf{0}
\end{aligned}
$$

and prove the following result:

Theorem 4.1. The solution to (4.2)-(4.4) with $\chi=0$ satisfies the following estimate for all $T \geq 0$ :

$$
\begin{aligned}
& \|\boldsymbol{u}\|_{l^{\infty}\left(0, T ; L^{2}\right)}^{2}+\frac{1}{2}\|\sqrt{v} \nabla \boldsymbol{u}\|_{l^{2}\left(0, T ; L^{2}\right)}^{2}+\Delta t\|p\|_{L^{2}\left(-\frac{\Delta t}{2}, T-\frac{\Delta t}{2}, A\right)} \\
& \leq\left\|\boldsymbol{u}_{0}\right\|_{L^{2}}^{2}+\frac{1}{2} \Delta t\left\|\sqrt{v} \nabla \boldsymbol{u}_{0}\right\|_{L^{2}}^{2}+\Delta t^{2}\left\|p_{0}\right\|_{A^{2}}^{2} .
\end{aligned}
$$

Proof. Taking the inner product of (4.5) with $2 \Delta t \boldsymbol{u}^{n+1}$, we obtain

$$
\begin{aligned}
& \left\|\boldsymbol{u}^{n+1}\right\|_{L^{2}}^{2}+\left\|\boldsymbol{u}^{n+1}-\boldsymbol{u}^{n}\right\|_{L^{2}}^{2}+\frac{1}{2} \Delta t\left\|\sqrt{v^{n+\frac{1}{2}}} \nabla \boldsymbol{u}^{n+1}\right\|_{L^{2}}^{2} \\
& \quad+\frac{1}{2} \Delta t\left\|\sqrt{v^{n+\frac{1}{2}}} \nabla\left(\boldsymbol{u}^{n+1}+\boldsymbol{u}^{n}\right)\right\|_{L^{2}}^{2}+2 \Delta t\left(\nabla p^{*, n+\frac{1}{2}}, \boldsymbol{u}^{n+1}\right) \\
& =\left\|\boldsymbol{u}^{n}\right\|_{L^{2}}^{2}+\frac{1}{2} \Delta t\left\|\sqrt{v^{n+\frac{1}{2}}} \nabla \boldsymbol{u}^{n}\right\|_{L^{2}}^{2} .
\end{aligned}
$$

For $\chi=0$, we deduce from the (4.3) and (4.4) that $\left(p^{n+\frac{1}{2}}-p^{n-\frac{1}{2}}\right) \in D(A)$ solves the following problem:

$$
a\left(p^{n+\frac{1}{2}}-p^{n-\frac{1}{2}}, q\right)=-\Delta t^{-1}\left(\nabla \cdot u^{n+1}, q\right), \quad \forall q \in D(A) .
$$

Taking $q=2 \Delta t^{2} p^{*, n+\frac{1}{2}}:=2 \Delta t^{2}\left(2 p^{n-\frac{1}{2}}-p^{n-\frac{3}{2}}\right)$ in the above and using the fact that $a(\cdot, \cdot)$ is a symmetric and coercive in $H^{1}(\Omega)$, we infer

$$
\begin{aligned}
& -2 \Delta t\left(\nabla \cdot \boldsymbol{u}^{n+1}, p^{*, n+\frac{1}{2}}\right) \\
& =2 \Delta t^{2} a\left(\delta p^{n+\frac{1}{2}}, 2 p^{n-\frac{1}{2}}-p^{n-\frac{3}{2}}\right) \\
& =2 \Delta t^{2} a\left(\delta p^{n+\frac{1}{2}}, p^{n+\frac{1}{2}}\right)+2 \Delta t^{2} a\left(\delta p^{n+\frac{1}{2}},-\delta p^{n+\frac{1}{2}}+\delta p^{n-\frac{1}{2}}\right) \\
& =\Delta t^{2}\left(\left\|p^{n+\frac{1}{2}}\right\|_{A}^{2}-\left\|p^{n-\frac{1}{2}}\right\|_{A}^{2}+\left\|\delta p^{n-\frac{1}{2}}\right\|_{A}^{2}-\left\|\delta^{2} p^{n+\frac{1}{2}}\right\|_{A}^{2}\right) .
\end{aligned}
$$

Subtracting (4.8) at time $t^{n}$ from (4.8) at time $t^{n+1}$, and taking the inner product of the resulting equation with $\Delta t \delta^{2} p^{n+\frac{1}{2}}$, we find

$$
\begin{aligned}
& \Delta t\left\|\delta^{2} p^{n+\frac{1}{2}}\right\|_{A}^{2}=-\left(\nabla \cdot\left(\boldsymbol{u}^{n+1}-\boldsymbol{u}^{n}\right), \delta^{2} p^{n+\frac{1}{2}}\right)=\left(\boldsymbol{u}^{n+1}-\boldsymbol{u}^{n}, \nabla \delta^{2} p^{n+\frac{1}{2}}\right) \\
& \leq\left\|\boldsymbol{u}^{n+1}-\boldsymbol{u}^{n}\right\|_{L^{2}}\left\|\nabla \delta^{2} p^{n+\frac{1}{2}}\right\|_{L^{2}} .
\end{aligned}
$$


Then using the coercivity property of the bilinear form $a(\cdot, \cdot)$, we infer

$$
\Delta t\left\|\nabla \delta^{2} p^{n+\frac{1}{2}}\right\|_{L^{2}}\left\|\delta^{2} p^{n+\frac{1}{2}}\right\|_{A} \leq\left\|\boldsymbol{u}^{n+1}-\boldsymbol{u}^{n}\right\|_{L^{2}}\left\|\nabla \delta^{2} p^{n+\frac{1}{2}}\right\|_{L^{2}}
$$

which implies $\Delta t^{2}\left\|\delta^{2} p^{n+\frac{1}{2}}\right\|_{A}^{2} \leq\left\|\boldsymbol{u}^{n+1}-\boldsymbol{u}^{n}\right\|_{L^{2}}^{2}$. Inserting this bound in (4.9), we obtain

$$
\Delta t^{2}\left(\left\|p^{n+\frac{1}{2}}\right\|_{A}^{2}+\left\|\delta p^{n-\frac{1}{2}}\right\|_{A}^{2}-\left\|p^{n-\frac{1}{2}}\right\|_{A}^{2}\right) \leq-2 \Delta t\left(\nabla \cdot \boldsymbol{u}^{n+1}, p^{*, n+\frac{1}{2}}\right)+\left\|\boldsymbol{u}^{n+1}-\boldsymbol{u}^{n}\right\|_{L^{2}}^{2} .
$$

Combining the above and (4.7), dropping some unnecessary terms, we arrive at

$$
\begin{aligned}
& \left\|\boldsymbol{u}^{n+1}\right\|_{L^{2}}^{2}+\frac{1}{2} \Delta t\left\|\sqrt{v^{n+\frac{1}{2}}} \nabla \boldsymbol{u}^{n+1}\right\|_{L^{2}}^{2}+\Delta t^{2}\left\|p^{n+\frac{1}{2}}\right\|_{A}^{2} \\
& \leq\left\|\boldsymbol{u}^{n}\right\|_{L^{2}}^{2}+\frac{1}{2} \Delta t\left\|\sqrt{v^{n+\frac{1}{2}}} \nabla \boldsymbol{u}^{n}\right\|_{L^{2}}^{2}+\Delta t^{2}\left\|p^{n-\frac{1}{2}}\right\|_{A^{2}}^{2} .
\end{aligned}
$$

Finally, we obtain the desired result by summing over $n$.

\section{Numerical Results and Discussions}

In this section, we present numerical experiments to verify the stability and accuracy of the proposed schemes.

Example 5.1. We consider the linearized Navier-Stokes equations (2.1) with the exact solution

$$
\begin{aligned}
& u(x, y, t)=\pi \sin t\left(\sin 2 \pi y \sin ^{2} \pi x,-\sin 2 \pi x \sin ^{2} \pi y\right), \\
& p(x, y, t)=\sin t \cos \pi x \sin \pi y .
\end{aligned}
$$

We have implemented the spectral direction splitting scheme (2.16)-(2.21), and for the sake of comparison, the corresponding non-splitting version (2.2)-(2.4) with $\chi=1 / 2$.

In the computations reported herein, we take $N=64$ so that the spatial discretization errors are negligible compared with the time discretization errors.

In Fig. 1, we plot, in a log-log scale, the $L^{2}$-velocity errors (the left figure) and the $H^{1}$-velocity errors (the right figure) at $T=1$. The $L^{2}$-pressure errors are presented in Fig. 2. The figures show that our algorithm is of second-order accuracy in the $L^{2}$-norm for the velocity, almost second-order for the $H^{1}$-norm of the velocity and for the $L^{2}$-norm of the pressure. The results by the non-splitting version are slightly better but with the same order of accuracy.

Example 5.2. We consider the exact solution of the linearized Navier-Stokes equations with variable viscosity (4.1) to be

$$
\begin{aligned}
& u(x, y, t)=\pi \sin t\left(\sin 2 \pi y \sin ^{2} \pi x,-\sin 2 \pi x \sin ^{2} \pi y\right), \\
& p(x, y, t)=\sin t \cos \pi x \sin \pi y, \\
& v(x, y, t)=\left(\sin ^{2} t+1\right)(x+2)(y+2) .
\end{aligned}
$$



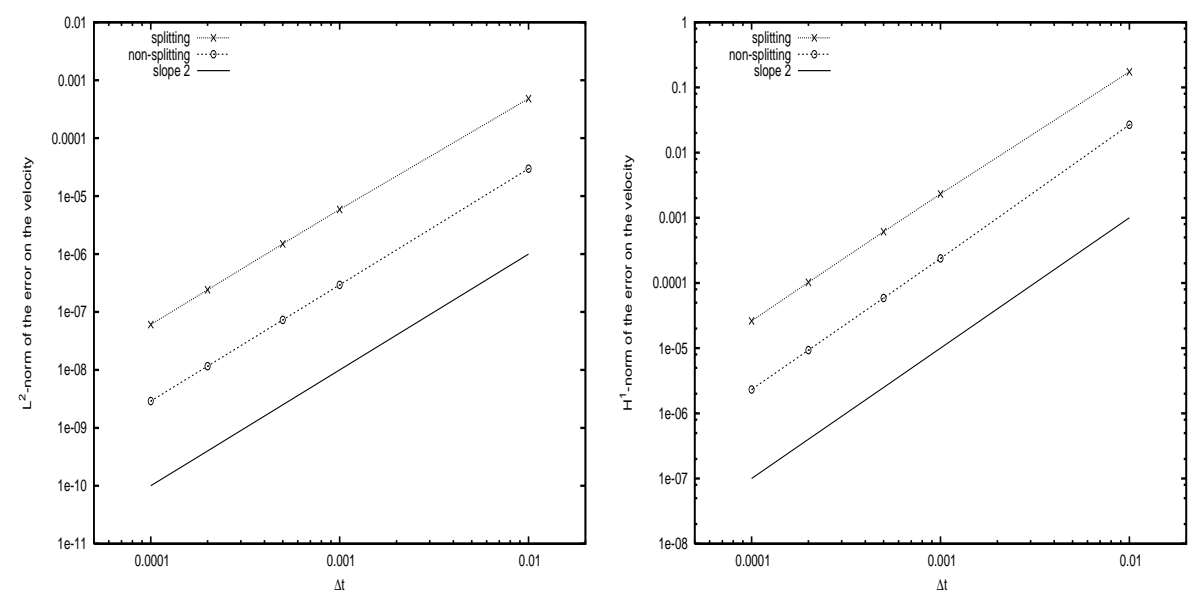

Figure 1: Left: Velocity error in $L^{2}$-norm; Right: Velocity error in $H^{1}$-norm.

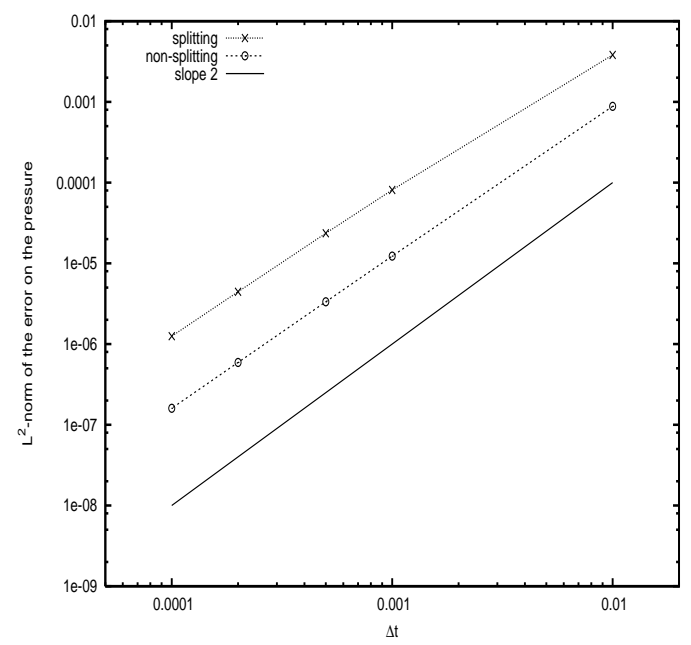

Figure 2: Pressure error in $L^{2}$-norm at $T=1$.

We use the scheme (4.2)-(4.4) discretized with the Legendre-spectral method similar to (2.16)-(2.21), and we take $N=128$ for all computations in this example.

The velocity errors in the $L^{2}$-norm and the $H^{1}$-norm at $T=1$ are shown in Fig. 3, while the pressure errors in the $L^{2}$-norm are shown in Fig. 4. We observe that the errors behave similarly as in the first example.

Notice that we used the space $P_{N}$ for both the velocity and pressure approximations in the scheme (2.16)-(2.21). It is well known that $P_{N} \times P_{N}$ does not satisfy the inf-sup condition (cf. for instance [1]). Although our spectral direction splitting scheme, is uniquely solvable without the inf-sup condition, the accuracy of the pressure approximation may still be affected by the lack of inf-sup condition as other projectional type schemes (cf. [5]). This can be checked by looking at the pressure convergence rate in $N$ with a fixed but very 

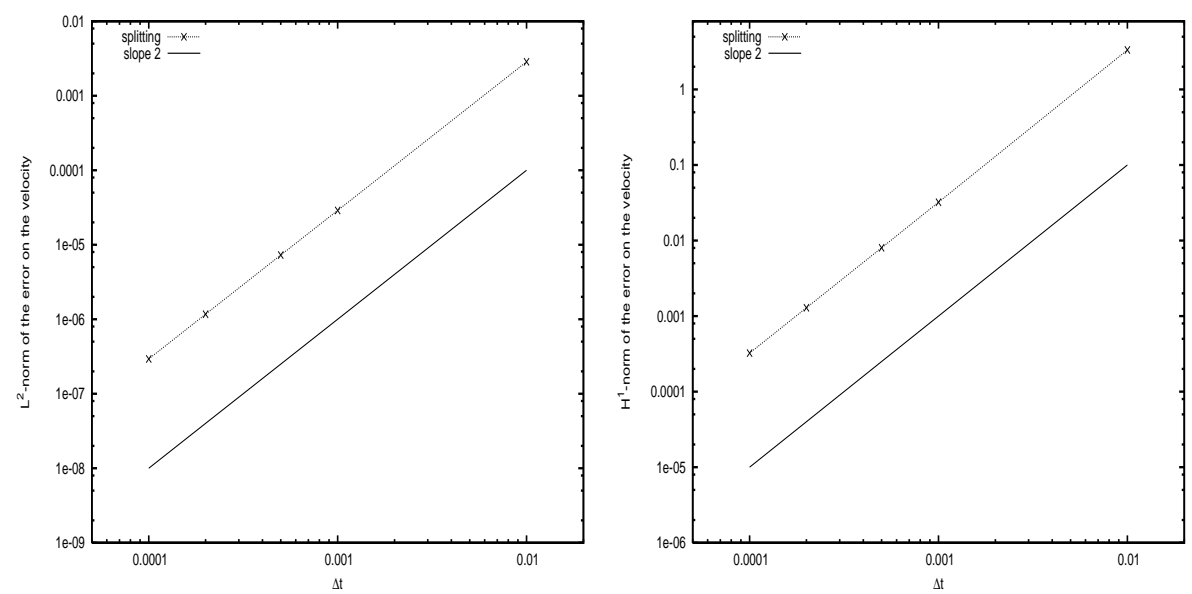

Figure 3: $L^{2}$-norm (left) and $H^{1}$-norm (right) error on the velocity at $T=1$.

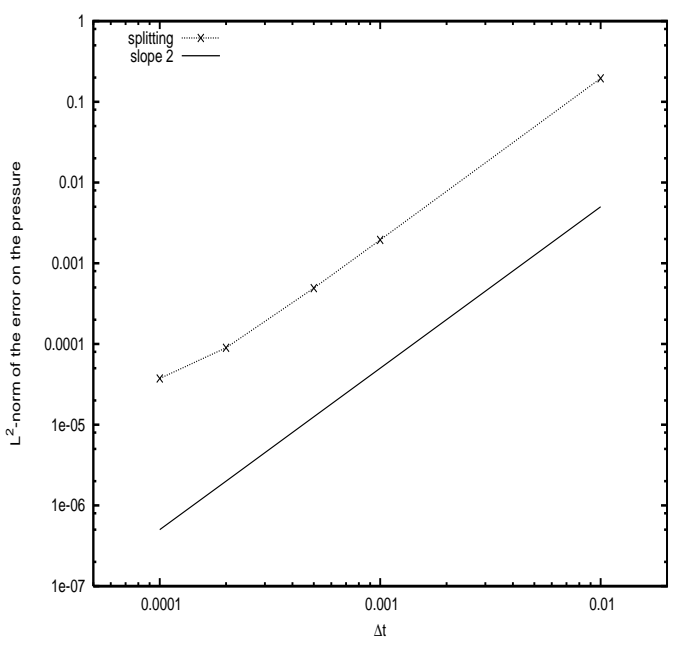

Figure 4: $L^{2}$-norm error on the pressure at $T=1$.

small time step. We show in Fig. 5 in log-log scale the error on the pressure measured in the $L^{2}$-norm as a function of the polynomial degree $N$ at $T=1$ with the time step $\Delta t=10^{-4}$. It is observed that the pressure approximation converges algebraically in $N$, instead of exponentially which is typical for spectral methods to smooth solutions. This indicates that the lack of inf-sup condition does affect the pressure convergence rate when $\Delta t \ll 1$. However, the velocity convergence rate is not affected.

\subsection{Concluding remarks}

We considered in this paper spectral direction splitting schemes for the incompressible Navier-Stokes equations. We constructed a hybrid of Legendre-collocation and LegendreGalerkin methods for the space variables and a pressure-stabilization/direction splitting 


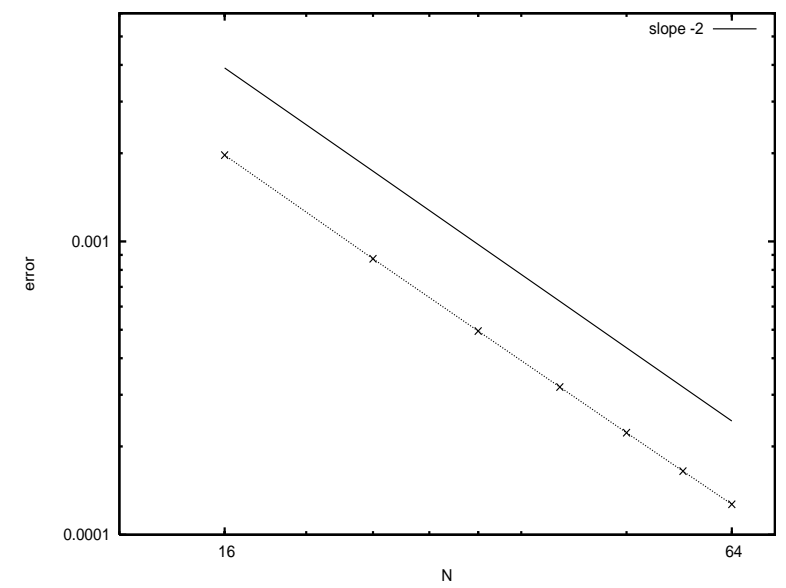

Figure 5: Effect of the inf-sup condition: pressure error vs. $N$ in log-log scale.

scheme for the temporal discretization, leading to a sequence of one-dimensional elliptic equations at each time step while preserving the same-order of accuracy as the usual pressure-stabilization schemes. We showed that these schemes were unconditionally stable. We also constructed a direction splitting scheme for the Navier-Stokes equations with variable viscosity.

The proposed schemes are very efficient and have the same order of accuracy as their non-splitting versions. They are particularly suitable for problems with variable viscosity and for parallel computing.

\section{Acknowledgments}

The work of J. Shen is partially supported by the NSF grant DMS-0915066. The research of C. Xu was partially supported by National NSF of China (Grant number 11071203).

\section{References}

[1] C. Bernardi and Y. Maday. Approximations Spectrales de Problèmes aux Limites Elliptiques. Springer-Verlag, Paris, 1992.

[2] Alexandre Joel Chorin. Numerical solution of the Navier-Stokes equations. Math. Comp., 22:745-762, 1968.

[3] J. L. Guermond and Jie Shen. On the error estimates for the rotational pressure-correction projection methods. Math. Comp., 73(248):1719-1737 (electronic), 2004.

[4] Jean-Luc Guermond and Peter D. Minev. A new class of fractional step techniques for the incompressible Navier-Stokes equations using direction splitting. C. R. Math. Acad. Sci. Paris, 348(9-10):581-585, 2010.

[5] J.L. Guermond, P. Minev, and Jie Shen. An overview of projection methods for incompressible flows. Comput. Methods Appl. Mech. Engrg., 195:6011-6045, 2006.

[6] J.L. Guermond, Minev P.D., and Salgado A.J. Convergence analysis of a class of massively parallel direction splitting algorithms for the Navier-Stokes equations. Preprint, 2011. 
[7] D. W. Peaceman and H. H. Rachford, Jr. The numerical solution of parabolic and elliptic differential equations. J. Soc. Indust. Appl. Math., 3:28-41, 1955.

[8] Jie Shen. On error estimates of projection methods for the Navier-Stokes equations: secondorder schemes. Math. Comp, 65:1039-1065, July 1996.

[9] R. Témam. Sur l'approximation de la solution des équations de Navier-Stokes par la méthode des pas fractionnaires. I. Arch. Rational Mech. Anal., 32:135-153, 1969.

[10] L. J. P. Timmermans, P. D. Minev, and F. N. Van De Vosse. An approximate projection scheme for incompressible flow using spectral elements. Int. J. Numer. Methods Fluids, 22:673-688, 1996. 